\title{
COMPARING LEARNING OBJECTS FOR EFFECTIVE LEARNING IN MATHEMATICS
}

\author{
L. Descalço, P. Carvalho \\ University of Aveiro (PORTUGAL)
}

\begin{abstract}
The high number of students per class, situation typically found in higher education in Portugal, makes it difficult to adopt an approach in which the student's work is seen as the core of the learning process. The use of digital contents in this context is helpful for the students to build their own appropriate environment outside the classroom, using all support and learning material created for that purpose. A fundamental problem we have in Mathematics is that many students are not well prepared in the previous concepts.

We have been developing and using several kinds of learning objects (LO) for calculus in the recent years, some of them dedicated to recall previous concepts. Among all kinds of contents, students valorize learning objects that simultaneously challenge them and give them useful feedback.

In this paper, we compare the usefulness of different kinds of LO using computers, in a calculus course for students of several sciences and engineering programs. For that purpose, we applied a survey, which was answered by $40 \%$ of a population of 290 students of the previously mentioned programs.

The learning objects include multiple-choice questions with detailed solutions, quizzes, formative assessment tests, short videos, and others. We present a quantitative analysis comparing different kinds of LO, considering students of several areas of study.
\end{abstract}

Together with the quantitative analysis, we add some qualitative considerations based on students' answers to open questions concerning the learning methodology adopted.

Keywords: Learning, computers, calculus, videos, multiple choice, self-diagnosis.

\section{INTRODUCTION}

Active learning is a process that consists of short course-related individual or small-group activities in class that students are encouraged to do, alternating with instructor-led intervals in which students' responses are processed and new information is provided. In a more informal way, anything that a teacher might ask students to do, such as answer questions in class, complete assignments and projects outside of class, or anything else other than being passively in a classroom can be considered as active learning. The high number of students per class, situation typically found in higher education in Portugal, makes it difficult to adopt an approach in which the student work is seen as the centre of the learning process. The use of digital contents in this context is helpful for the students to build their own appropriate environment outside the classroom, using all the support and learning material for that purpose. A fundamental problem we have in Mathematics is that many students are not well prepared in previous concepts.

Since 1995 ([1]), many materials have been developed to perform active learning pedagogies together with new methods for delivering course materials. Although active learning can be seen simply as integrating in-class activities instead of traditional exposition, some teachers have been adopting other strategies and models such as flipped classroom by adding some instructional contents to work outside the classroom ([2]). Students are required to view these contents before classes. According to Tucker ([3]) students should use the time in class to work on problems, advance concepts, and engage in collaborative learning.

As a learning methodology, we adopted an approach to engage students that consists in a direct computer based individual learning outside the classroom together with learning activities in the classroom.

It is part of our previous work, the creation and use of computer systems and digital contents to provide the best possible conditions for independent and autonomous learning in Calculus courses. 
We have been working for several years in SIACUA (stands for Interactive Computer Learning System, University of Aveiro, in Portuguese) (see [4]), a system for independent study with parameterized multiple-choice questions ([5]) with detailed solutions, and other Learning Objects such as small videos, among others, providing feedback computed by a Bayesian user model ([6]). These systems, containing several kinds of learning objects, are described in [7].

The concept of Learning Objects is grounded in the object-oriented paradigm of computer science [8]. The main goal of Learning Objects is the creation of instructional components that can be reused numerous times in different learning contexts. Therefore, Learning Objects are entities, digital or nondigital, that can be used, reused, or referenced during learning features supported by technology. They can be of several types and include multimedia contents, instructional software and software tools that are referenced during technology-supported learning. Some examples include digital pictures, animation and video clips, small pieces of text, applications available via Web, like GeoGebra and Wolfram Alpha, for instance, which are very well known and used in Math subjects. In SIACUA we integrate reusable digital resources, including Web pages that combine text, pictures and small videos, as well as a platform dedicated to self-assessment and formative assessment with immediate feedback in qualitative and quantitative form, that offers a complete educational experience.

There are several benefits for students because of the implementation of flexible teaching and learning options. In fact, flexible approaches to teaching and learning are particularly useful for workers and students who have family commitments, or those who for any reason are unable to attend classes at set times on a regular basis. Likewise, all students have more choices about how, when, and where they learn and flexible approaches to teaching and learning can be used to facilitate workplace learning, as well as other off-classes experiences.

During this research, along with online activities, specially using SIACUA, we used other motivating elements for learning, in particular, challenging problems to be solved by students as homework individually or in group.

In SIACUA, we use several kinds of Learning Objects. In addition to pictures and quizzes, we emphasize multiple-choice questions with detailed solutions and small videos.

Multiple-choice questions are challenging and time-consuming to create. In a simple way, a multiplechoice question is composed of two parts: a question text identifying the question or problem, and a set of possible answers or alternatives. These contain the correct answer to the question, and several distractors that are plausible but incorrect answers to the question. Students respond to the question by choosing the alternative that they believe to be the correct answer. There are many advantages in using this type of questions for assessment. One advantage is that the questions are easy to mark and can even be scored by a computer, which makes them an attractive assessment approach for large classes. Well-designed multiple-choice questions allow testing for a breadth of content and goals and provide an objective measurement of students' abilities. We can point out some good characteristics that must be taken into consideration when one elaborates multiple-choice questions:

- Use familiar language, in the sense that the question should employ the same terminology that is used in the course; in particular, students are likely to dismiss distractors with unfamiliar terms as incorrect.

- The instructor should avoid giving verbal association clues between the question and the possible answers; if the correct choice uses words that are very similar to words found in the text of the question, students are more likely to pick it as the correct answer.

- Avoid trick questions. Questions should be designed so that students who know the material can find the correct answer. This is better than designing questions to lead students to an incorrect answer, by misleading phrasing or emphasizing an otherwise unimportant detail of the solution.

- Avoid negative sentences. Students often fail to observe negative wording and it can confuse them. As a result, even students who are familiar with the course material often make mistakes on negatively worded questions.

- The full problem should be stated in the question text. A good multiple-choice question allows students to answer the question without looking at the options.

- All relevant material should be in the question text rather than repeated in each of the alternative's information; this makes options simpler to read and understand, making it easier for students to answer the question quickly. 
- Eliminate excessive wording and irrelevant information, since these confuse students and lead them to waste time.

To perform and design the alternatives, some good practices are:

- The number of alternatives should be limited to three to five options per question. Four-item choices are the most used, but the three or five choice items are just as effective, especially because it is difficult to come up with plausible distractors.

- We must be sure that there is only one correct answer. The situations in which other options are partially correct should be avoided. The distractors should be incorrect answers to the question.

- The distractors should be appealing and plausible. If the distractors are unnatural or nonsensical, students will locate the correct answer too easily, even if they have little knowledge. In addition, the distractors should be kept similar in length and type of language as the correct solution and should represent common mistakes made by students.

- Choices must be grammatically consistent with the text question.

- Avoid using "all of the above" as well as "none of the above". If students know two of the options are correct, the answer must be "all of the above" and if they know one is incorrect, the answer must not be "all of the above". On the other hand, the option "none of the above" does not test whether the student knows the correct answer, it tests only that the student knows the distractors are not correct.

- Most students know that few things are universally true or false, so distractors with the words "always", "never", "all", or "none" can often be easily dismissed and should be avoided in the distractors.

- Avoid overlapping choices. Make the alternatives mutually exclusive. It should never be the case that if one of the distractors is true, another distractor must be true as well.

The multiple-choice questions we use are parameterized questions. Hence, we create question generation templates that produce several similar questions. This parameterization is achieved by using Python programming language, together with useful packages, including MEGUA (see [9]), which means there are no limitations for question creation. Another innovative feature is that questions have detailed solutions, also parameterized. A question presents four choices, from a set that can contain more than four items. Hence, the detailed solution does not explain why each of the incorrect choices is false since that would not make sense for nondisplayed choices. Instead, the detailed solution provides a general explanation from which students can understand which one is the true statement and why the other three are false. This is in fact a clever way to teach, since students are challenged to think and select an option and only after an explanation is presented. We believe that this aspect may be one of the reasons that, as we will see, makes students value this resource so much (see [5]).

In [10] is proposed a conceptual framework for enhancing engagement from educational videos that identifies online activities as a type of self-regulated learning. This kind of work requires students to monitor their own self-discipline and to identify learning difficulties. Some research ([11]) shows that a video is a more engaging way to present new and complex material to students and stimulates students to be interested in learning more about the topic. In fact, videos have become an important instructional tool in higher education. They can be integrated as part of traditional courses, as we do in this work, and can serve as a cornerstone of blended courses.

The first and most important guideline for maximizing student attention to educational video is to keep it short. Other recommendations about the use of videos in a learning context are:

- Videos must be brief and targeted on learning goals.

- Videos and other visual elements are appropriate parts of an explanation; these elements must be complementary rather than redundant.

- Short videos can be used to highlight important ideas or concepts.

- It is important to use a conversational, enthusiastic style more than a too formal one to enhance engagement.

- We should embed videos in a context of active learning by using somehow guiding questions, interactive elements, or even associated homework assignments. 
In this experiment, we used this kind of instructional material to test its effectiveness in the learning process of calculus' students. This paper describes the implementation of Learning Objects as part of flexible learning and teaching in a second year Engineering Calculus course.

\section{METHODOLOGY}

Many factors influence the performance and success of students in an advanced Calculus course. Some of those factors include previous knowledge, students' motivation, study skills and the methodology implemented by teachers. The data presented in this paper is based on a semester involving 290 students from several Engineering programs. The methodology is blended learning in which online learning activities take place even if it sometimes directs students to offline tasks. Students work individually or in group and the teacher provides face-to-face support in classes or individual tutoring. We are essentially interested in comparing the value students attribute to the several kinds of Learning Objects and learning activities implemented. This comparison includes six issues and is based on a survey taken by 118 of the 290 students, that is about $40 \%$ of the total.

We start by describing the six issues included in this blended learning methodology and present the results of the survey.

1 Lists with challenging problems for homework.

Each element of this list consists of a set of challenging problems that must be solved as homework. Afterwards, a short quiz is performed in class about one random problem from the same set. The main goal of these elements is to keep students interested and working during the semester as well as investigate autonomously and develop searching skills.

2 Multiple-choice questions with detailed solutions in SIACUA.

These questions are available during the whole semester. Students can think about the problem for a while and then try to choose the correct option. They also have the possibility to see a proposed detailed solution without choosing any option. This solution is automatically shown if a wrong option is selected. Answers are used by the system to compute the Bayesian model beliefs about the student's knowledge.

3 Progress bars in SIACUA.

Progress bars in SIACUA show the beliefs about the students' knowledge and can be useful for self-diagnosis. These bars are clickable and present questions about the corresponding topic.

4 The three learning elements per topic.

Each study topic in SIACUA has three clickable elements: one for recalling previously needed topics, the second for a short introduction to the topic and a third containing references for a complete study. These elements consist of web pages dynamically built, including text, pictures, and embedded videos.

5 Short videos in SIACUA with introductions and examples.

These videos consist of screen capture with the teacher's voice and are mainly aimed for a first contact with the topic. They are short, with an average duration between 3 and 5 minutes.

6 Mini tests with multiple-choice questions.

These tests consist of multiple-choice questions from SIACUA, performed in the assessment platform PmatE ([12]). This platform is very well known since it is used in the yearly national competitions with thousands of students from all over the country. It allows their users to immediately see correct answers and their marks.

The survey consists essentially of six questions, asking students to attribute a value between 1 and 10 for the importance of the corresponding element in the learning process. Additionally, there is an open question that allows students to make suggestions to improve these tools.

\section{RESULTS}

Figure 1 presents a boxplot showing the distribution of the answers from all the 118 students to the six questions in the survey. 


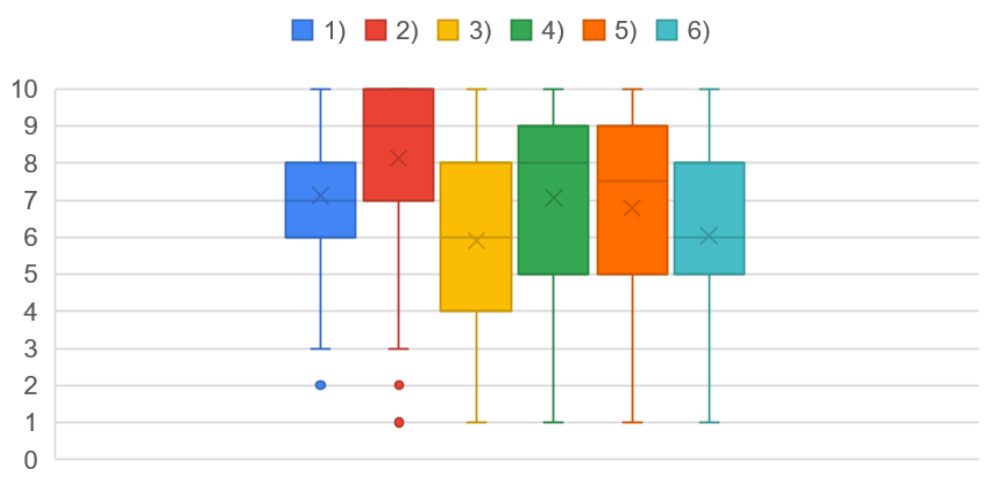

Figure 1. Answers to survey from all courses.

The distribution of answers is clear with the average marked with a cross and the median with a horizontal line. We can see the results are positive for all elements, the multiple-choice questions with detailed solution are the most valued resource and progress bars together with PmatE mini tests are the less valued elements.

Figure 2 presents the results only from the Industrial Management Engineering (IME) program.

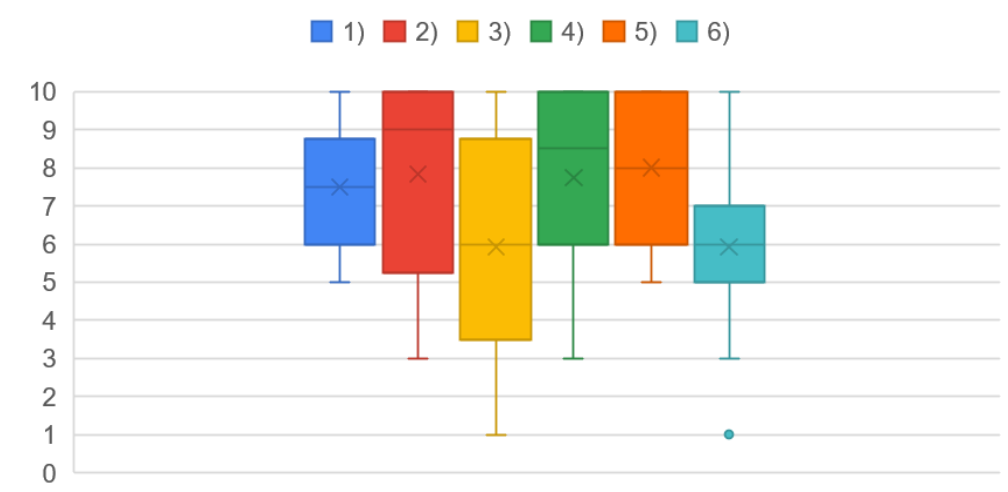

Figure 2. Answers from Industrial Management Engineering program.

Finally, Figure 3 presents results from the Mechanical Engineering (ME) program.

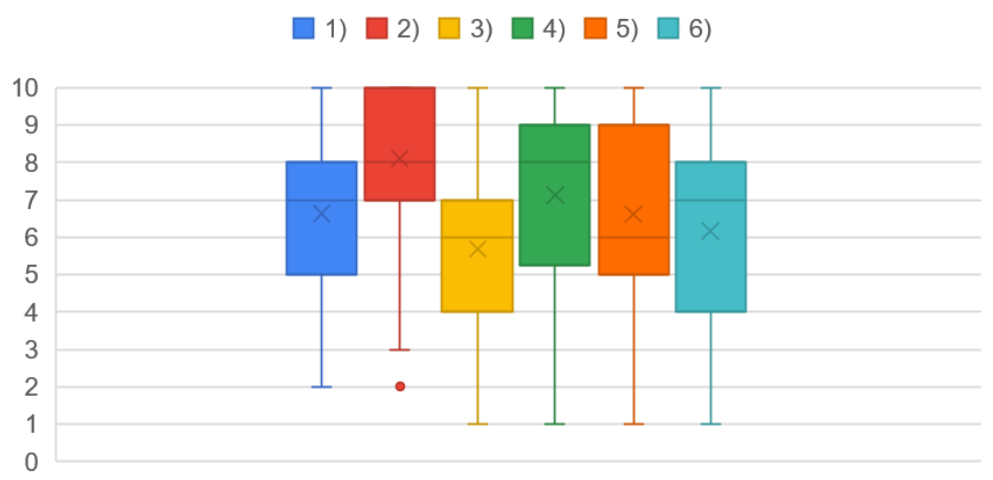

Figure 2. Answers from Mechanical Engineering program.

We distinguish these two cases to emphasize the influence of teacher attitude in the value students attribute to resources. The IME teacher is the author of the three learning elements per topic (4) and videos (5) and the ME teacher is the author of lists of problems (1) and multiple-choice questions with detailed solutions (2). 


\section{CONCLUSIONS}

The most surprising conclusion is that students consider multiple-choice questions with detailed solutions the most important resource for studying, even when compared with the small videos. Multiple-choice questions are typically used for assessment or self-diagnosis. However, we used them here essentially as Learning Objects in a learn-by-example approach and as a clever way to provide explanations. Detailed answers are the immediate way to get an answer to a specific question that arises in a certain context and at a certain time.

On the other hand, in [13] Postareff defends that there is an interactive relationship between teachers' and students' emotions and behavior. In fact, teachers' emotions and empathy are contagious and can influence the learning outcomes of their students. This is true both for positive emotions such as enjoyment, pride, excitement, and negative emotions like anxiety or frustration. Positive teachers' emotions and the pleasure of doing Learning Objects to provide meaningful learning to their students can promote student enjoyment and enthusiasm of learning.

This is particularly visible in this experience where the preferences of the students who answered to the survey are in line with the objects produced by the teacher who shares the face-to-face activities in the classroom.

There are many different suggestions in the open question of the survey. But one conclusion is clear: the students value our work and ask for more Learning Objects in the topics that are not yet fully covered. Our innovative way of providing explanations by using parameterized multiple-choice questions with a detailed solution is greatly valued. This makes us believe that the cost-benefit of this work is favourable which motivates us to proceed with the production of digital contents, to improve the learning platform SIACUA and to use the teaching methodology adopted in general.

\section{ACKNOWLEDGEMENTS}

This work is supported by The Center for Research and Development in Mathematics and Applications (CIDMA) through the Portuguese Foundation for Science and Technology (FCT Fundação para a Ciência e a Tecnologia), references UIDB/04106/2020 and UIDP/04106/2020.

\section{REFERENCES}

[1] R. S. Grabinger and J. C. Dunlap, "Rich environments for active learning: a definition," ALT-J Research in Learning Technology, vol. 3, no. 2, pp. 5-34, 1995.

[2] J. Bishop and M.A. Verleger, "The flipped classroom: A survey of the research," in ASEE National Conference Proceedings, Atlanta, GA., 2013.

[3] B. Tucker, "The flipped classroom," Education Next, vol. 12, no. 1, pp. 82-83, 2012.

[4] SIACUA - Sistema Interativo de Aprendizagem por Computador - Universidade de Aveiro. Retrieved from https://siacua.web.ua.pt/

[5] P. Oliveira, P. Carvalho, "Mathematics Exercise Generator: The Language of Parameterized Exercises", in Developing Technology Mediation in Learning Environments (Soares, F., Lopes, A. P., Brown, K., \& Uukkivi, A. Ch. , eds) 15, pp. 264-282, Hershey, PA: IGI Global, 2020.

[6] E. Millán, L. Descalço, G. Castillo, P. Oliveira and S. Diogo, "Using Bayesian networks to improve knowledge assessment", Computers and Education, vol. 60, no. 1, pp. 436-447, 2013.

[7] P. Carvalho P. Oliveira and L. Descalço, "Motivating Study Before Classes on Flipped Learning", Proceedings of EDULEARN18 Conference, IATED, 6295-6300, Palma, Spain, 2018.

[8] V.S. Freeman, "Learning objects in microbiology: A new resource". Clinical Laboratory Science, 17(2), 80, 2004.

[9] P. Cruz, P. Oliveira and D. Seabra, "Exercise templates with Sage", Tbilisi Mathematical Journal, vol. 5(2), 37-44, 2012.

[10] D.L. Schacter, K.K. Szpunar, "Enhancing attention and memory during video-recorded lectures". Sch Teach Learn Psychol.,1: 60-71, 2015. 
[11] B.R.Stockwell, M.S. Stockwell, M. Cennamo, E. Jiang, "Blended learning improves science education". Cell., 162, 933-936, 2015.

[12] PmatE - Projeto Matemática Ensino - Universidade de Aveiro. Retrieved from https://pmate.ua.pt

[13] L. Postareff and S. Lindblom-Ylänne, "Emotions and confidence within teaching in higher education", Studies in Higher Education, 36:7, 799-813, 2011.

DOI: 10.1080/03075079.2010.483279 